Article

\title{
Clematichinenoside (AR) Attenuates \\ Hypoxia/Reoxygenation-Induced H9c2 \\ Cardiomyocyte Apoptosis via \\ a Mitochondria-Mediated Signaling Pathway
}

\author{
Haiyan Ding ${ }^{1,2}$, Rong Han ${ }^{2}$, Xueshan Chen ${ }^{2}$, Weirong Fang ${ }^{1}$, Meng Liu ${ }^{3}$, Xuemei Wang ${ }^{4}$, \\ Qin Wei ${ }^{4}$, Nandani Darshika Kodithuwakku ${ }^{5}$ and Yunman Li ${ }^{1, *}$ \\ 1 State Key Laboratory of Natural Medicines, Department of Physiology, China Pharmaceutical University, \\ Nanjing 210009, China; dinghaiyan79@163.com (H.D.); weirongfang@163.com (W.F.) \\ 2 College of Pharmacy, Xinjiang Medical University, Urumqi 830011, China; ronger051125@163.com (R.H.); \\ chenxueshan2010@sina.com (X.C.) \\ 3 Cancer Hospital Affiliated to Xinjiang Medical University, Urumqi 830011, China; \\ liuxiaomeng198154@163.com \\ 4 Xinjiang Key Laboratory of Medical Animal Model Research, \\ Clinical Medical Research Institute of the First Affiliated Hospital of Xinjiang Medical University, \\ Urumqi 830011, China; wxuemei1983@sina.com (X.W.); fashion1027@163.com (Q.W.) \\ 5 Institute of Indigenous Medicine, University of Colombo, Rajagiriya 11600, Sri Lanka; darshi_ko@yahoo.com \\ * Correspondence: yunmanlicpu@hotmail.com; Tel.: +86-25-8327-1173
}

Academic Editors: Atanas G. Atanasov, Karel Šmejkal and Elke Heiss

Received: 11 April 2016; Accepted: 20 May 2016; Published: 30 May 2016

\begin{abstract}
Mitochondria-mediated cardiomyocyte apoptosis is involved in myocardial ischemia/reperfusion (MI/R) injury. Clematichinenoside (AR) is a triterpenoid saponin isolated from the roots of Clematis chinensis with antioxidant and anti-inflammatory cardioprotection effects against $\mathrm{MI} / \mathrm{R}$ injury, yet the anti-apoptotic effect and underlying mechanisms of AR in MI/R injury remain unclear. We hypothesize that AR may improve mitochondrial function to inhibit MI/R-induced cardiomyocyte apoptosis. In this study, we replicated an in vitro H9c2 cardiomyocyte MI/R model by hypoxia/reoxygenation $(\mathrm{H} / \mathrm{R})$ treatment. The viability of $\mathrm{H} 9 \mathrm{c} 2$ cardiomyocytes was determined by MTT assay; apoptosis was evaluated by flow cytometry and TUNEL experiments; mitochondrial permeability transition pore (mPTP) opening was analyzed by a calcein-cobalt quenching method; and mitochondrial membrane potential $(\Delta \Psi \mathrm{m})$ was detected by JC-1. Moreover, we used western blots to determine the mitochondrial cytochrome $\mathrm{c}$ translocation to cytosolic and the expression of caspase-3, Bcl-2, and Bax proteins. These results showed that the application of AR decreased the ratio of apoptosis and the extent of $\mathrm{mPTP}$ opening, but increased $\triangle \Psi \mathrm{m}$. AR also inhibited $\mathrm{H} / \mathrm{R}$-induced release of mitochondrial cytochrome $\mathrm{c}$ and decreased the expression of the caspase-3, Bax proteins. Conversely, it remarkably increased the expression of Bcl-2 protein. Taken together, these results revealed that $\mathrm{AR}$ protects $\mathrm{H} 9 \mathrm{c} 2$ cardiomyocytes against $\mathrm{H} / \mathrm{R}$-induced apoptosis through mitochondrial-mediated apoptotic signaling pathway.
\end{abstract}

Keywords: clematichinenoside (AR); apoptosis; H9c2 cardiomyocytes; hypoxia/reoxygenation; mitochondria; signaling pathway

\section{Introduction}

Ischemia/reperfusion is associated with thrombolysis, angioplasty and coronary bypass surgery which relieve myocardial ischemia, but simultaneously cause further damage to myocardial tissue, which is known as myocardial ischemia/reperfusion (MI/R) injury [1]. MI/R injury is one of the 
death threats in various cardiovascular diseases [2]. During MI/R injury, cardiomyocytes undergo apoptosis [3,4], which results in myocardial contractile dysfunction, compensatory hypertrophy and reparative fibrosis, all of which further increase myocardial injury, and ultimately leads to cardiac dysfunction [5]. Apoptosis is considered one of the key pathological processes in MI/R injury and the amount of apoptosis determines the severity of MI/R injury [6]. Therefore, deciphering the mechanisms by which the cardiomyocytes apoptosis in MI/R injury has an important impact on the development of effective therapeutics [7,8].

Apoptosis is a process of programmed cell death and can be induced by several signaling pathways, in which the mitochondrial pathway plays an important role and mediates the apoptotic modulation in the whole process [6]. Mitochondrial-mediated apoptotic pathway can be triggered by several factors. One is the opening of mitochondrial permeability transition pore (mPTP), a large, nonselective conductance pore located in the inner mitochondrial membrane. $\mathrm{mPTP}$ exerts a crucial effect in triggering apoptosis of cardiomyocytes exposed MI/R injury [9]. Once the MPTP is open, mitochondrial membrane potential $(\Delta \Psi \mathrm{m})$ is depolarized, leading to pro-apoptotic factors leakage cascade reactions and mitochondrial dysfunction. Cytochrome $c$, one of the pro-apoptotic factors, has been regarded a decisive step to initiate apoptosis in MI/R injury [10]. The leakage of cytochrome c promotes caspase activation, in which caspase- 3 is the key caspase responsible for promoting cell death [11]. Additionally, Bcl-2 family proteins affect the permeability of the mitochondrial membrane in MI/R-induced apoptosis [12]. Therefore, ameliorating mitochondrial function is an effective therapeutic strategy to reduce apoptosis and protect the heart from MI/R injury.

Clematichinenoside (AR, Figure 1, 3-O-[ $\alpha$-L-rhamnopyranosyl-( $1 \rightarrow 6)-\beta$-D-glucopyranosyl- $(1 \rightarrow 4)$ $\beta$-D-glucopyranosyl-( $1 \rightarrow 4)$ - $\beta$-D-ribopyranosyl-( $(\rightarrow 3)-\alpha$-L-rhamnopyranosyl-( $1 \rightarrow 2)-\alpha$-L-arabinopyranosyl]oleanolic acid 28-O-[ $\alpha$-L-rhamnopyranosyl-( $1 \rightarrow 4)-\beta$-D-glucopyranosyl-( $1 \rightarrow 6)-\beta$-D-glucopyranosyl] ester), is a major bioactive component isolated from the roots of Clematis chinensis, whose isolation procedure has been discussed by Liu et al. [13]. Clematichinenoside has anti-arthritic and anti-inflammatory effects, and can attenuate myocardial infarction following MI/R injury [14-16]. However, whether AR has anti-apoptotic effects on MI/R-induced cardiomyocyte apoptosis remains unexplored. We hypothesized that AR may improve the mitochondria function to inhibit MI/R-induced cardiomyocyte apoptosis. Therefore, the aims of the present study were to investigate whether AR can indeed inhibit MI/R-induced cardiomyocytes apoptosis and to decipher the mechanisms underlying the mitochondrial-mediated apoptotic signaling pathway.

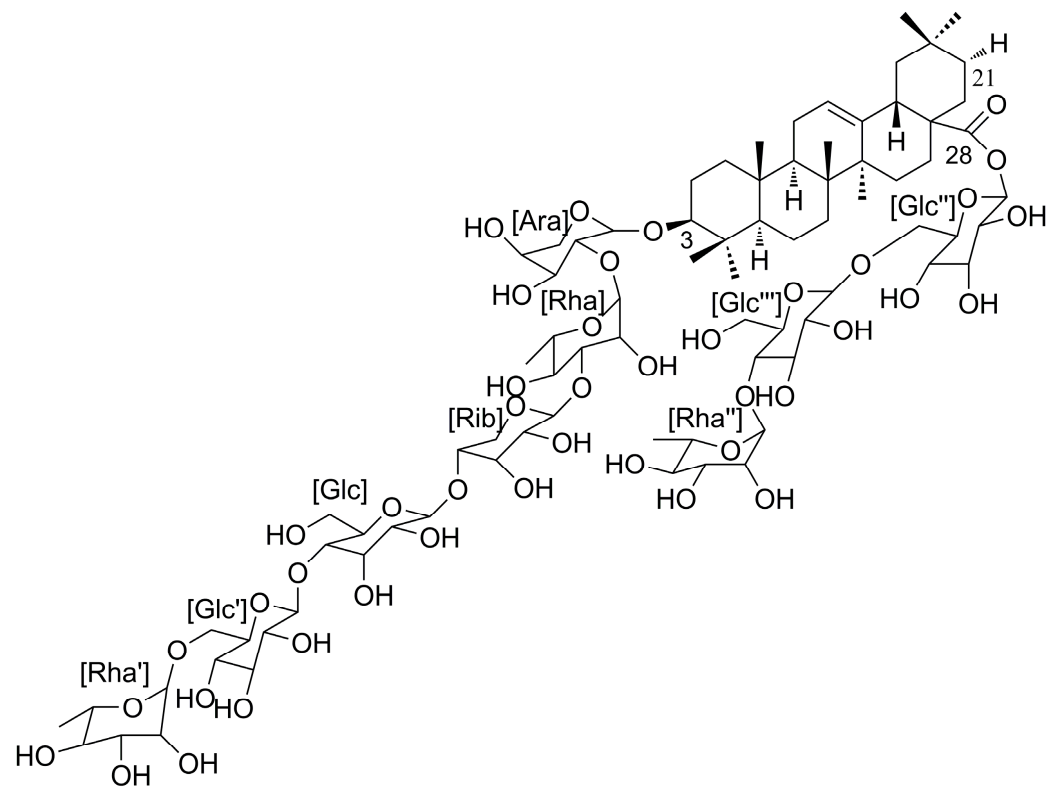

Figure 1. Chemical structure of clematichinenoside (AR). 


\section{Results}

\subsection{AR Pretreatment Increases Cell Viability}

Viable cells were reduced with $\mathrm{H} / \mathrm{R}$ treatment and accounted for $47.1 \%$ of the control group. Compared with $\mathrm{H} / \mathrm{R}$ group, cell viability was increased significantly in $\mathrm{AR}(1,10$, $100 \mu \mathrm{M}$ ) pretreatment groups from $47.1 \%$ to $61.7 \%, 72.6 \%$ and $85.4 \%$, respectively (Figure 2). The results suggested that AR owned protective effect in $\mathrm{H} 9 \mathrm{c} 2$ cardiomyocytes against $H / R$ injury in a concentration-dependent manner.

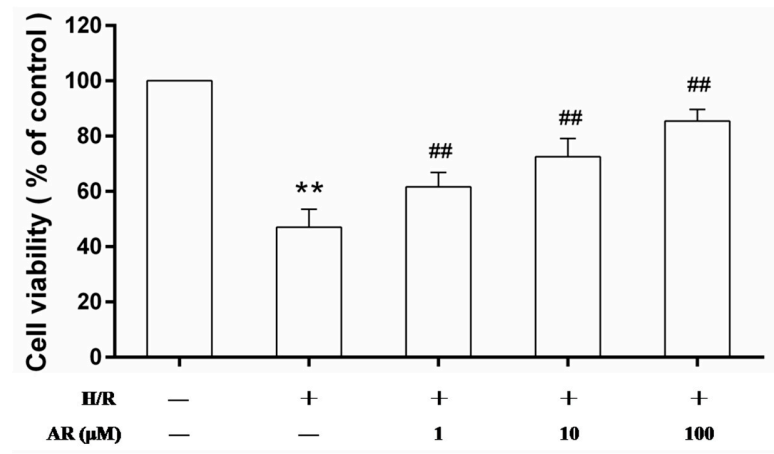

Figure 2. Effects of $A R$ on $H 9 c 2$ cardiomyocytes viability. After treatment with $A R(1,10,100 \mu M)$ for $24 \mathrm{~h}, \mathrm{H} 9 \mathrm{c} 2$ cardiomyocytes were treated with $\mathrm{H} / \mathrm{R}$ injury. Cells viability was analyzed by [3-(4,5-dimethylthiazol-2-yl)-2,5-diphenyltetrazolium bromide] (MTT) assay, and the value of the control group was considered $100 \%$. Values presented are mean $\pm \operatorname{S.D}(n=8)$. ${ }^{* *}: p<0.01$ vs. control group; ${ }^{\# \#}: p<0.01$ vs. H/R group.

\subsection{AR Pretreatment Inhibits H9c2 Cardiomyocytes Apoptosis against H/R Injury}

In order to investigate whether $\mathrm{AR}$ protects $\mathrm{H} 9 \mathrm{c} 2$ cardiomyocytes against $\mathrm{H} / \mathrm{R}$-induced apoptosis, cells were detected by Annexin V-FITC/PI double staining (Figure 3).
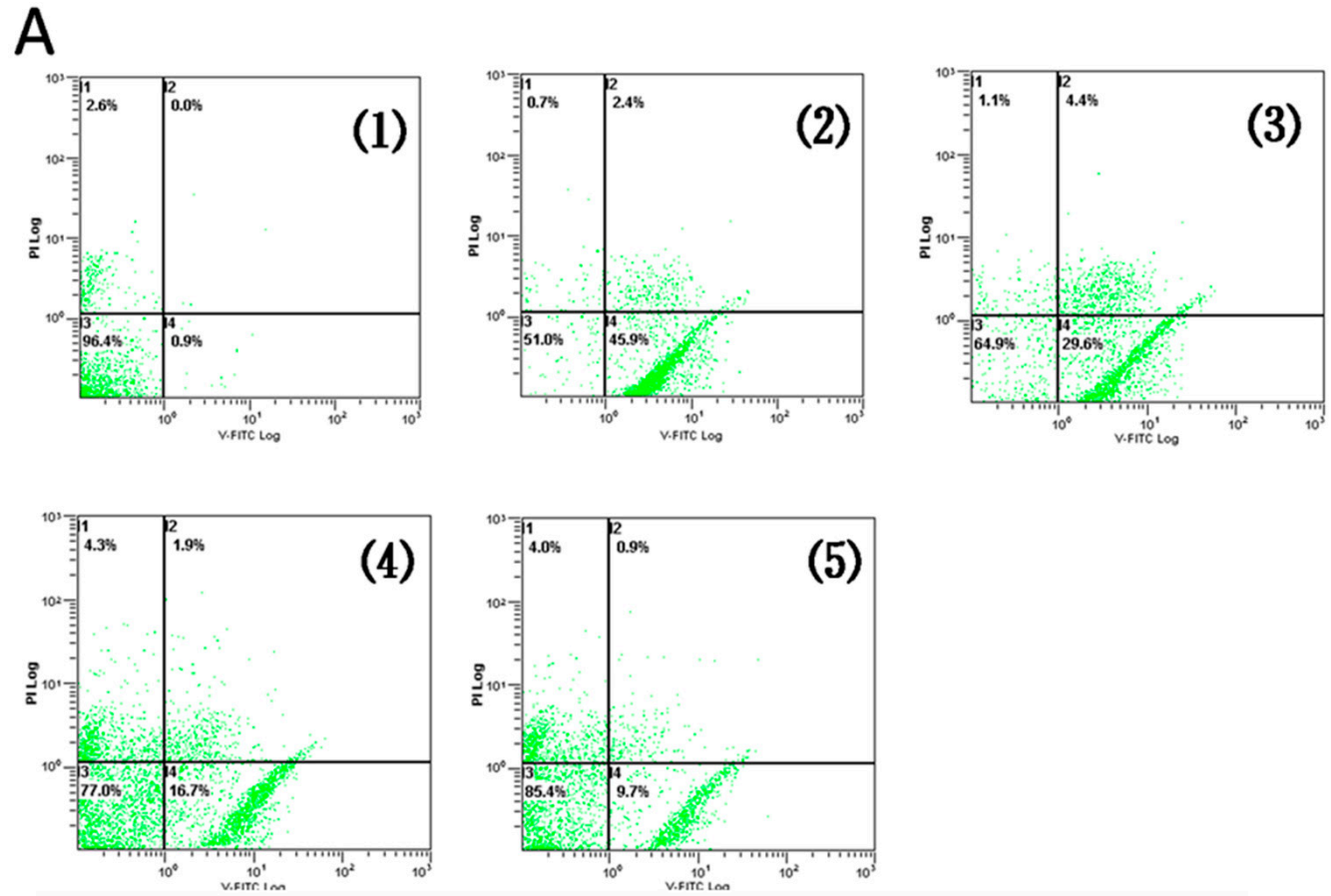

Figure 3. Cont. 


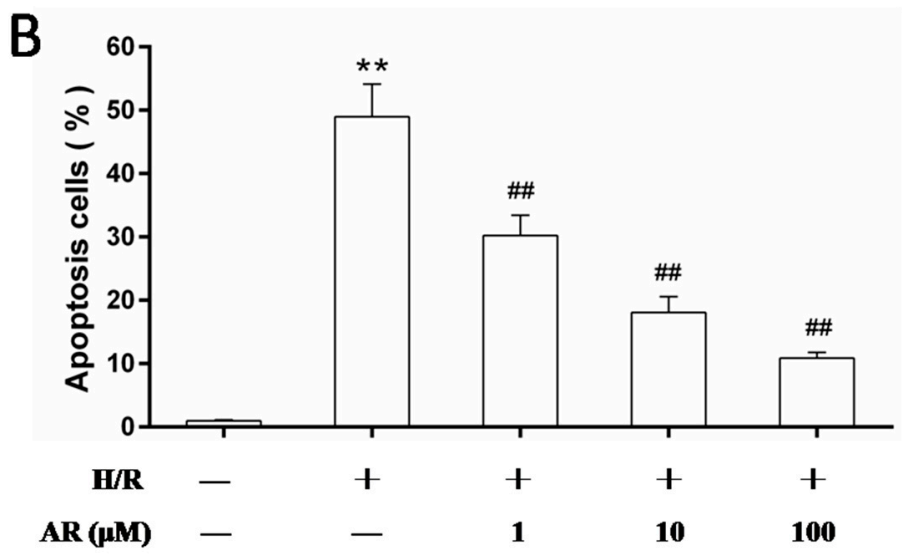

Figure 3. Effects of AR on H/R-induced H9c2 cardiomyocytes apoptosis. Flow cytometry analysis was carried out to determine apoptotic ratio in experimental groups. (A): (1) Control group; (2) H/R group; (3) AR low-dose $(1 \mu \mathrm{M})$ group; (4) AR intermediate-dose $(10 \mu \mathrm{M})$ group; (5) AR high-dose (100 $\mu \mathrm{M})$ group; (B) The histogram shows the relative proportion of apoptosis cells in different experimental groups. Values presented are mean \pm S.D $(n=5) .{ }^{* *}: p<0.01$ vs. control group; ${ }^{\# \#}: p<0.01$ vs. H/R group.

Only Annexin V-FITC positive but PI negative cells were considered as early apoptotic cells, which were defined as the quantity. Flow cytometry analysis results demonstrated that there was significant elevation of apoptosis in $\mathrm{H} / \mathrm{R}$ group compared to control $(49.0 \%$ vs. $0.98 \%, p<0.01)$. While apoptosis reduced remarkably in pretreatment with $\mathrm{AR}(1,10,100 \mu \mathrm{M})$ compared with $\mathrm{H} / \mathrm{R}$ group ( $p<0.01$, Figure 3B). The results showed pretreatment of AR inhibited H/R-induced apoptosis in $\mathrm{H} 9 \mathrm{c} 2$ cardiomyocytes.

\subsection{AR Pretreatment Protects $H 9 c 2$ Cardiomyocytes against $H / R$ Injury}

To further determine the protective role of AR on H/R-induced apoptosis in H9c2 cardiomyocytes, we observed cell morphology by nuclear morphology by DAPI and terminal deoxynucleoitidyl transferase-mediated biotinylated UTP nick end labeling (TUNEL) staining. As shown in Figure 4A, DAPI staining showed that regular contour and round or elliptical nuclei existed in normal cells.

\section{A}
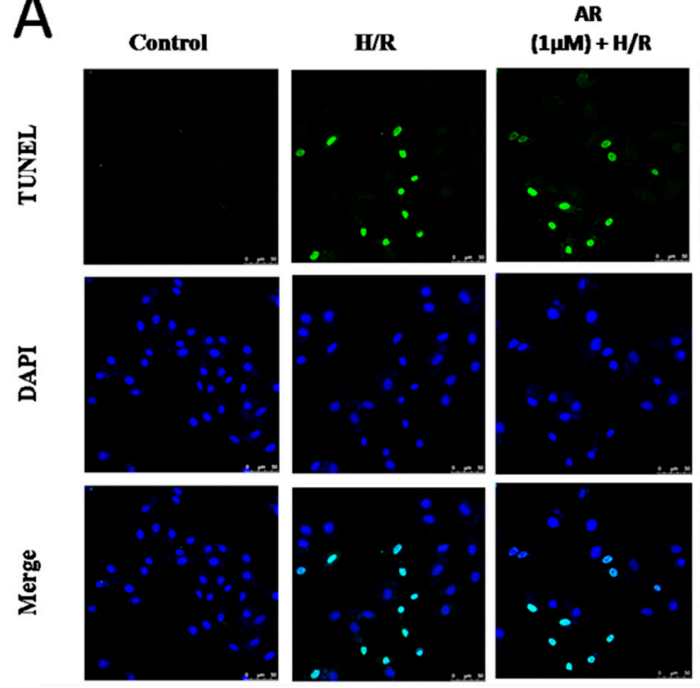
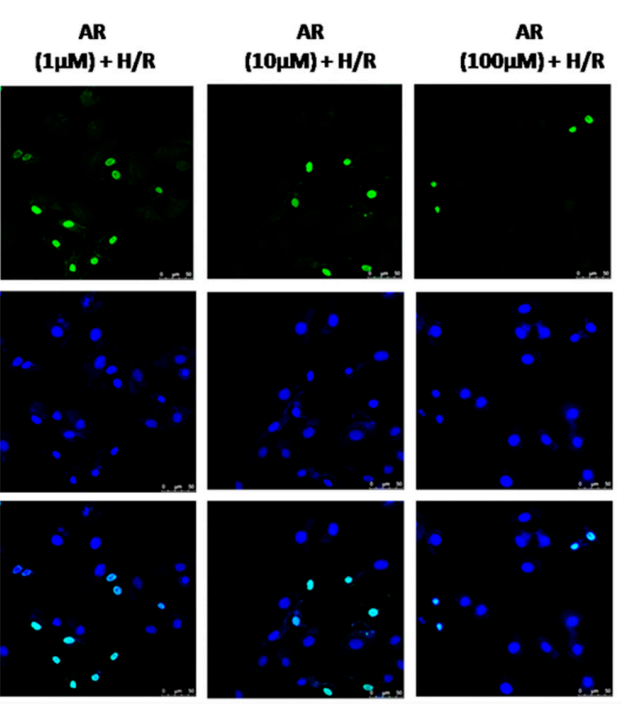

Figure 4. Cont. 


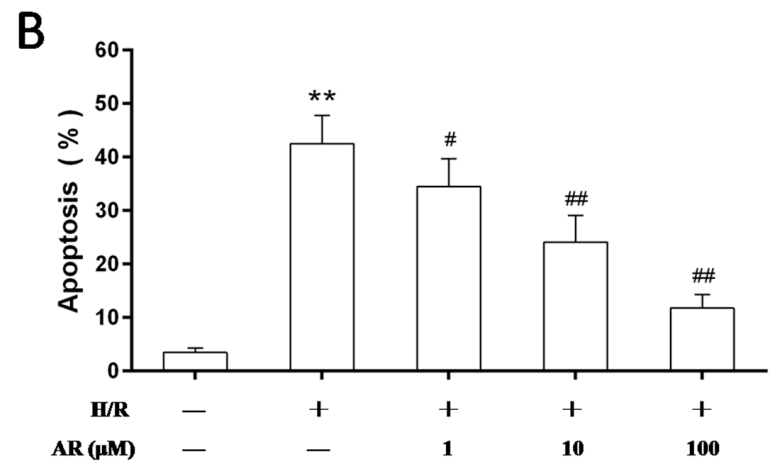

Figure 4. Protection effects of $\mathrm{AR}$ against $\mathrm{H} / \mathrm{R}$-induced $\mathrm{H} 9 \mathrm{c} 2$ cardiomyocytes apoptosis. Pretreatment with AR $(1,10,100 \mu \mathrm{M})$ for $24 \mathrm{~h}$ prior to $\mathrm{H} / \mathrm{R}$, morphology and apoptosis of H9c2 cardiomyocytes were assessed by DAPI and TUNEL staining. (A) Representative images of TUNEL-positive cells (green, first row) and DAPI counterstaining (blue, middle row). Scale bar: $50 \mu \mathrm{m}$; (B) The histogram shows the relative proportion of TUNEL-positive cells in experimental groups. Values are mean \pm S.D $(n=5) .{ }^{* *}: p<0.01$ vs. control group; ${ }^{\#}: p<0.05$ vs. H/R group; ${ }^{\# \#}: p<0.01$ vs. $\mathrm{H} / \mathrm{R}$ group.

In contrast, cells with H/R-treated appeared with smaller nuclei and shrunk chromatin. Pre-administration of AR significantly improved cell morphology and reduced the number of $\mathrm{H} / \mathrm{R}$-induced apoptosis. The apoptosis ratio was higher in H/R group compared to control group (42.5\% vs. $3.5 \%, p<0.01$, Figure 4B). Compared with H/R group, AR $(1,10,100 \mu \mathrm{M})$ decreased $\mathrm{H} / \mathrm{R}$-induced apoptosis from $42.5 \%$ to $34.5 \%, 24.1 \%$ and $11.8 \%(p<0.05, p<0.01$ and $p<0.01$ respectively). The results illustrated that AR possessed the effect not only protected cell morphology but also reduced $\mathrm{H} / \mathrm{R}$-induced apoptosis.

\subsection{AR Pretreatment Inhibits $m P T P$ Opening Induced by H/R Injury}

To investigate whether AR inhibits mitochondrial permeability transition pore (mPTP) opening, we detected the mPTP opening mode with the calcein-cobalt method. Compared with the control, we observed that fluorescence significantly decreased in H/R cells $(p<0.01)$. However, pretreatment of $\operatorname{AR}(1,10,100 \mu \mathrm{M})$ demonstrated a significant higher level compared with $\mathrm{H} / \mathrm{R}$ group $(p<0.05, p<0.01$ and $p<0.01$ respectively, Figure 5). These results demonstrated that AR owned the ability to reduce the extent of $\mathrm{mPTP}$ opening of $\mathrm{H} / \mathrm{R}$-injured cells in response to the protection effect of mitochondria.

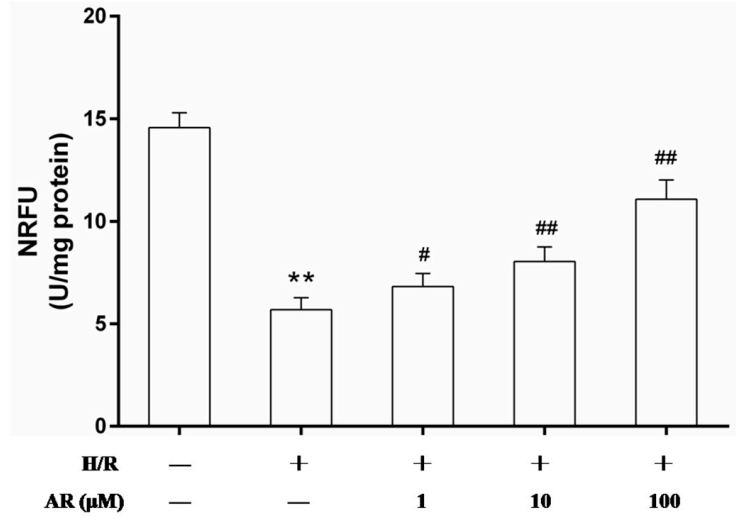

Figure 5. AR pretreatment inhibited the mPTP opening in H/R-injured $\mathrm{H} 9 \mathrm{c} 2$ cardiomyocytes. The mPTP opening was detected by the calcein-cobalt method. All experimental groups were measured the relative normalized fluorescent units (NRFU) of calcein. Values are mean $\pm \operatorname{S.D}(n=5) .{ }^{* *}: p<0.01$ vs. control group; : $p<0.05$ vs. H/R group; ${ }^{\#}: p<0.01$ vs. H/R group. 


\subsection{AR Pretreatment Maintains the Mitochondrial Membrane Potential ( $\Delta \Psi m)$ in H/R-Treated Cells}

To further assess the role of AR on the mitochondrial function, $\Delta \Psi \mathrm{m}$ was measured by using

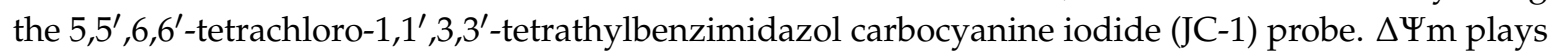
a critical function in cell life. Maintaining $\Delta \Psi \mathrm{m}$ is essential to preserve mitochondrial function. The collapse of the $\Delta \Psi \mathrm{m}$ is an important sign of apoptosis occurrence. As shown in Figure 6A, lower level of red fluorescence and higher level of green fluorescence were observed in H/R-treated cells, but pretreatment of $\operatorname{AR}(1,10,100 \mu \mathrm{M})$ reversed the fluorescence changes $(p<0.01)$. The $\Delta \Psi \mathrm{m}$ was calculated as the fluorescent ratio of red to green. The lower ratio illustrated the level of mitochondrial depolarization (Figure 6B). These results revealed that pretreatment of AR prevented the mitochondrial depolarization and maintained the mitochondrial intact. Moreover, it suggested that the protective role of AR against $\mathrm{H} / \mathrm{R}$-injured apoptosis associated with mitochondria function.
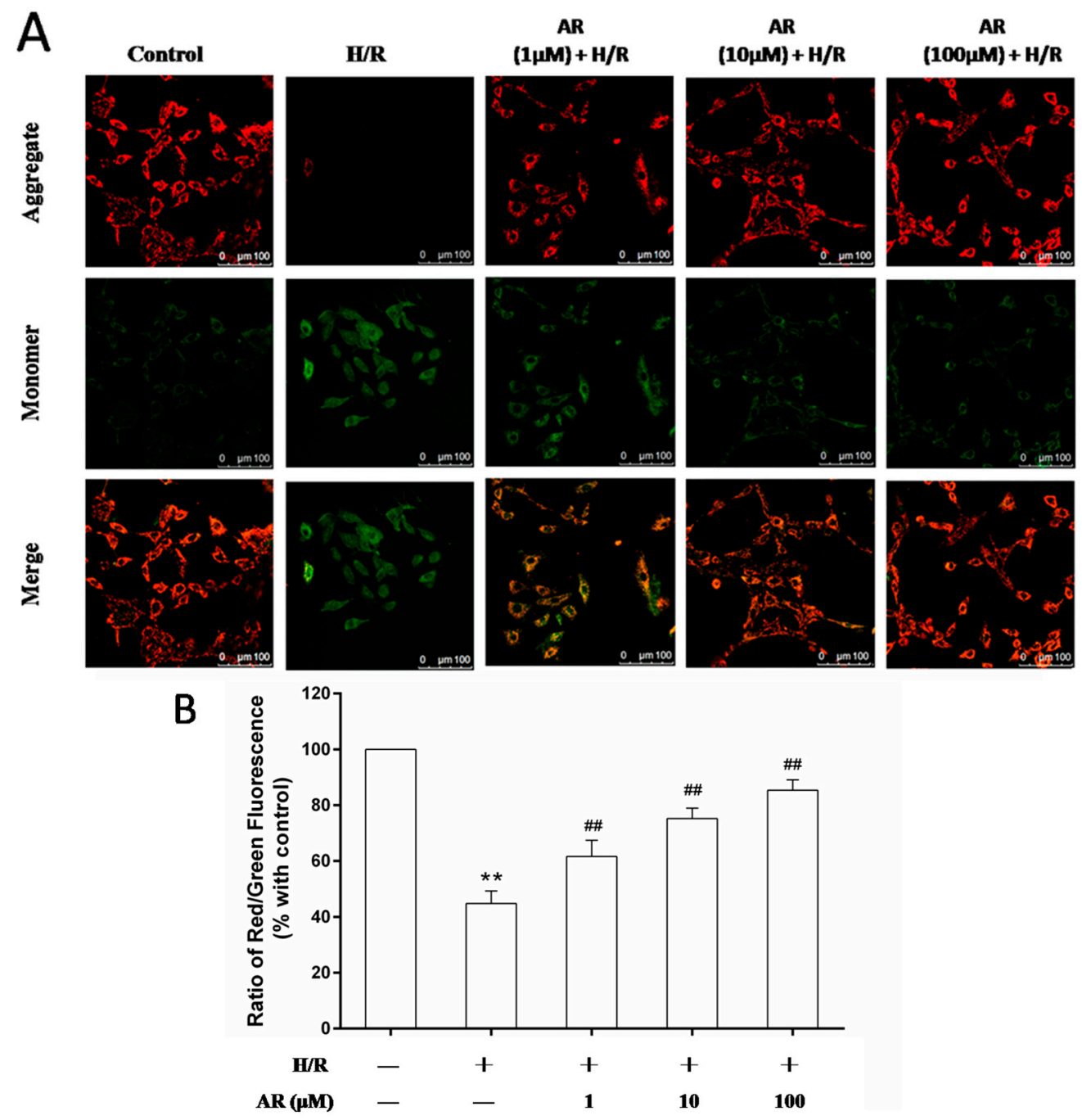

Figure 6. AR pretreatment maintained the $\Delta \Psi \mathrm{m}$ in $\mathrm{H} / \mathrm{R}$-injured cells. $\Delta \Psi \mathrm{m}$ of the different groups was estimated with JC-1 probe. For red fluorescent excitation is $585 \mathrm{~nm}$ and emission is $590 \mathrm{~nm}$; for green fluorescent excitation is $514 \mathrm{~nm}$ and emission is $530 \mathrm{~nm}$. (A) JC-1 aggregates red fluorescence in mitochondria of normal cells. JC-1 accrues as green fluorescence monomer in the cytosol of apoptotic cells, which indicates $\Delta \Psi \mathrm{m}$ collapsed. Merged images showed the co-localization of JC-1 aggregates and monomers. Scale bar: $100 \mu \mathrm{m}$; (B) The $\Delta \Psi \mathrm{m}$ of cells in different groups was calculated as the fluorescent ratio of red to green. Values are mean \pm S.D $(n=5) .{ }^{* *}: p<0.01$ vs. control group; $\#$ : $p<0.01$ vs. H/R group. 


\subsection{AR Pretreatment Prevents Cytochrome c Release}

Leakage of cytochrome $\mathrm{c}$ from mitochondria to cytosol is an important sign in cell apoptotic pathway after mPTP opening and $\triangle \Psi \mathrm{m}$ collapse. In the present study, cytochrome celeased from mitochondria was determined by western blot. Compared with the control, mitochondrial membranes damage was severe in $H / R$ group, leading to the leakage of cytochrome $c$ from mitochondria. There was significant decrease of cytochrome $\mathrm{c}$ in the mitochondria in H/R-treated cells $(p<0.01$, Figure 7A). However, cytochrome $\mathrm{c}$ in the mitochondria was increased significantly with pretreatment of $\operatorname{AR}(1,10,100 \mu \mathrm{M})$ compared with $\mathrm{H} / \mathrm{R}$ group $(p<0.05, p<0.01$ and $p<0.01$ respectively). Intriguingly, cytochrome $c$ was observed only in cytosol of $H / R, A R$ low-dose groups and intermediate-dose groups. Furthermore, there was significant difference between $\mathrm{H} / \mathrm{R}$ and AR low-dose, intermediate-dose groups $(p<0.05, p<0.01$, Figure $7 \mathrm{~B})$. These results showed that cytochrome $c$ release was efficiently inhibited by AR pretreatment in $H / R$-injured H9c2 cardiomyocytes, which revealed that AR may protect cardiomyocytes apoptosis potentially by regulating the mitochondrial apoptotic pathway.

A
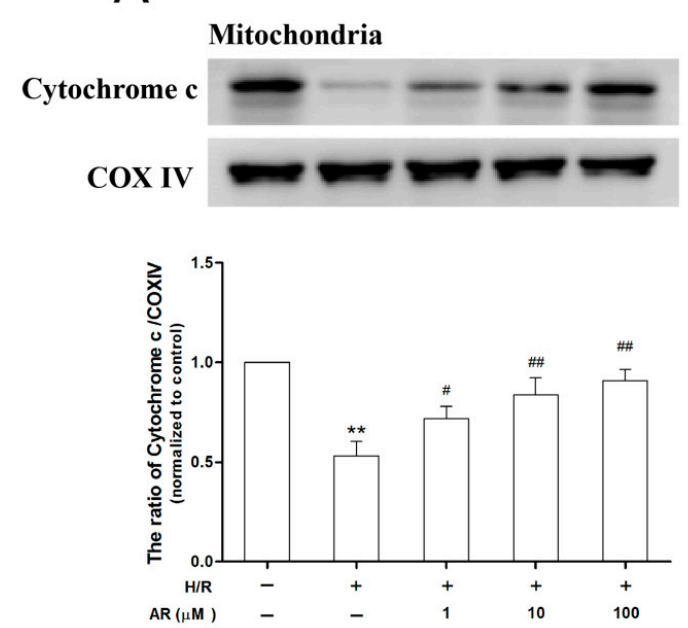

B
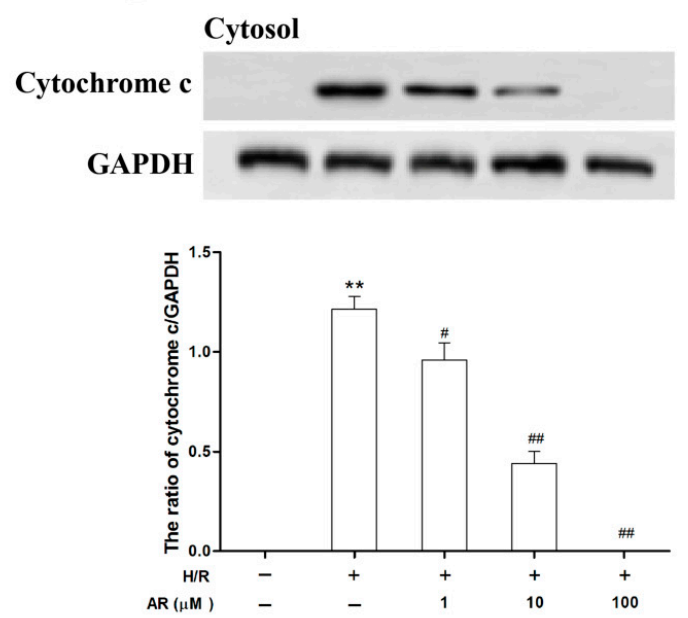

Figure 7. Effects of AR on preventing cytochrome c release in $\mathrm{H} 9 \mathrm{c} 2$ cardiomyocytes exposed to H/R. (A) The release of cytochrome c in mitochondria; (B) Cytosolic translocation of mitochondrial cytochrome $\mathrm{c}$ in different experimental groups. Values presented are mean \pm S.D $(n=3) .{ }^{* *}: p<0.01$ vs. control group; ${ }^{\#}: p<0.05$ vs. H/R group; ${ }^{\# \#}: p<0.01$ vs. H/R group.

\subsection{AR Pretreatment Suppresses Caspase-3 Activity and Increases the Ratio of Bcl-2 to Bax Exposed to $H / R$ Injury}

Caspase-3 plays a determinant role in apoptotic progress. Compared with the control, expression of caspases-3 was significantly promoted in H/R group $(p<0.01)$, while pretreatment of AR $(1,10$, $100 \mu \mathrm{M})$ reversed these increases significantly $(p<0.05, p<0.01$ and $p<0.01$ respectively, Figure 8A). These results demonstrated that the beneficial effects of AR were accompanied with the suppression of the caspases-3 activity in H/R-injured H9c2 cardiomyocytes.

Bcl-2 and Bax proteins, the Bcl-2 family proteins, serve important roles in triggering the mitochondrial death cascade. In the present study, the expression of Bcl-2 and Bax proteins were measured by western blot. As shown in Figure 8B, in $\mathrm{H} / \mathrm{R}$ group Bcl-2 expression decreased and Bax expression increased significantly compared with control group. However, pretreatment of AR $(1,10$, $100 \mu \mathrm{M})$ up-regulated Bcl-2 expression, suppressed Bax expression and enhanced the Bcl-2/Bax ratio significantly compared with the $\mathrm{H} / \mathrm{R}$ group $(p<0.05, p<0.01$ and $p<0.01$ respectively). These results suggested that AR protected against H/R-induced apoptosis in H9c2 cardiomyocytes by modulating the balance of Bcl-2 and Bax proteins. 
A

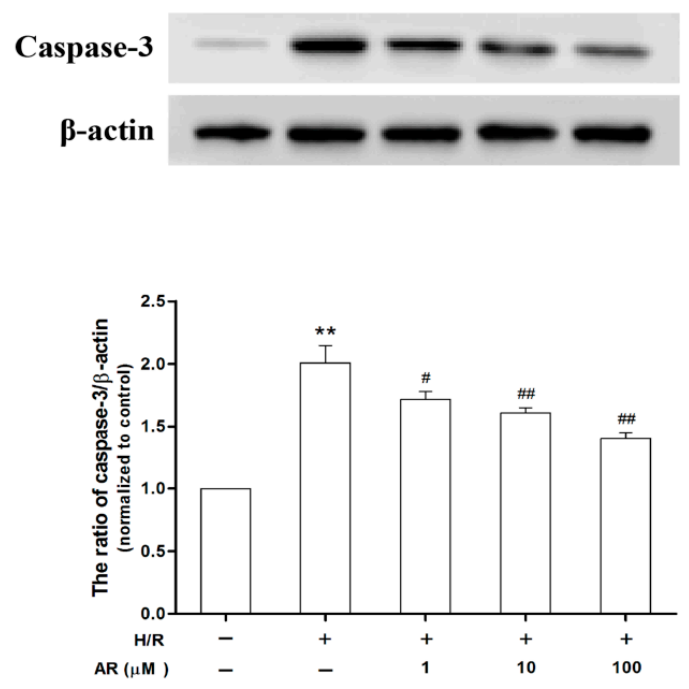

B
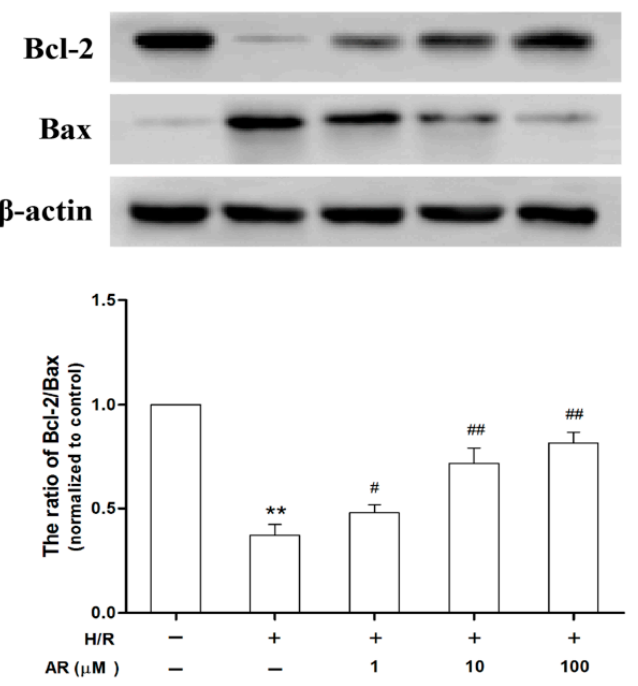

Figure 8. AR pretreatment suppressed the activity of casepase- 3 and enhanced the ratio of Bcl-2 to Bax in H9c2 cardiomyocytes subjected to H/R. (A) Casepase-3 expression of different groups; (B) The ratio of Bcl-2 to Bax in various experimental treatment groups. Values presented are mean \pm S.D $(n=3)$. **: $p<0.01$ vs. control group; $:$ : $p 0.05$ vs. H/R group; ${ }^{\# \# . ~} p<0.01$ vs. H/R group.

\section{Discussion}

It is well known that mitochondria not only control the energy metabolism of the body, but also regulate cell apoptosis or necrosis [17]. Increasing evidence suggests that MI/R-induced cell apoptosis is closely associated with mitochondria dysfunction [18,19]. Therefore, ameliorating the mitochondrial function is an effective method to attenuate the H/R-induced injury in cardiomyocytes. Based on these notions, we hypothesized that the cardioprotection of AR may connect with regulating mitochondrial function. Zhang et al. have reported that AR attenuates myocardial infarction in $\mathrm{H} / \mathrm{R}$ injury by its antioxidant effects and by restoring the balance of nitric oxide synthase [16]. Our previous study found that AR has anti-arthritic effects through PI3K/Akt signaling pathway and attenuating TNF- $\alpha$ associated collagen-induced arthritis [14]. Recently, we found that AR alleviates cerebral inflammatory injury through A20-NF- $\mathrm{kB}$ signal pathway [20]. The present study revealed that the application of AR increases the cell viability and reduces $H / R$-induced cardiomyocyte apoptosis through improving mitochondria function, demonstrated by inhibiting $\mathrm{mPTP}$ opening, maintaining mitochondrial permeability transition pore $(\Delta \Psi \mathrm{m})$, preventing cytochrome c leakage from mitochondria to cytoplasm, suppressing caspase-3 activity, and increasing the ratio of Bcl-2 to Bax exposed H/R injury. All of which are closely associated to mitochondria-dependent signaling pathways. These results further verified our previous hypothesis. To the best of our knowledge, the present study has elucidated for the first time that the mitochondria-mediated signaling pathway is one of the mechanisms underlying AR protects the H/R-injury induced cardiomyocyte apoptosis.

mPTP exerts a crucial role in triggering the apoptosis of cardiomyocytes exposed H/R injury [9]. Once $\mathrm{mPTP}$ is open, mitochondrial membrane becomes sensitive, resulting in dissipation of membrane potential, that in turn affecting the role of mitochondria [21]. $\Delta \Psi \mathrm{m}$ is another important factor regulating mitochondrial function, and acts as an indicator of the status of mPTP [22]. Intriguingly, we showed that AR significantly alleviates the H/R injury-induced mPTP opening and $\Delta \Psi \mathrm{m}$ depolarization in a dose-dependent manner. This result is consistent with the finding that lycopene exerts its anti-apoptotic effect through suppressing MPTP opening to inhibit mitochondrial dysfunction [23].This suggests that cardioprotection of AR during H/R injury is tightly associated with normal function of mitochondria. 
During $\mathrm{H} / \mathrm{R}$ injury, along with $\mathrm{mPTP}$ opening and $\Delta \Psi \mathrm{m}$ depolarization mitochondrial dysfunction is inevitable, and then pro-apoptotic factors, such as cytochrome c, are released, which has been considered as a decisive procedure to activate the mitochondrial apoptotic pathway [10]. Furthermore, the leakage cytochrome c promotes caspase activation, which executes and controls apoptotic signaling [24]. Among various caspases, caspase-3 is the key caspase and responsible for promoting cell death [11]. Consistent with these results, we indicated that increases of the leakage cytochrome $\mathrm{c}$ and caspase- 3 activity were detected in H/R treatment. However, this study showed that the leakage of cytochrome $c$ from mitochondria to cytoplasm and the activity of caspase- 3 were significantly attenuated by a pretreatment with AR dose-dependently. All these findings demonstrated that AR closely associates with the functional regulation of mitochondrial, through which preventing $\mathrm{H} / \mathrm{R}$ injury-induced cardiomyocyte apoptosis.

Bcl-2 family is a kind of apoptosis related genes, its expression and regulation are the key factors influencing apoptosis [25]. Bcl-2/Bax ratio is another important parameter in mitochondria signaling pathway. Alteration of the Bcl-2/Bax ratio influences apoptotic balance [26-28]. Bcl-2 family proteins play an important role in apoptosis by regulating the permeabilization of the mitochondrial membrane [12]. In our study, we had another novel discovery that AR can significantly increase Bcl-2 and decrease Bax protein expression, therefore enhancing the Bcl-2/Bax ratio in a concentration-dependent manner compared with H/R group. Bcl-2 regulates the opening and closing of mitochondrial permeability transition pore (mPTP) to exert an anti-apoptosis role, but Bax plays a pro-apoptosis function in the family. The evidence could indicate the up-regulation the ratio of $\mathrm{Bcl}-2 / \mathrm{Bax}$ in AR pretreatment group, which consists with our finding that AR protects cardiomyocytes against $\mathrm{H} / \mathrm{R}$ injury through mitochondrial pathway. Cook et al have reported that $\mathrm{Bcl}-2$ regulates $\mathrm{mPTP}$ opening in opposition to Bax, prevents cytochrome c leakage, suppresses caspase activity and decreases cell apoptosis $[29,30]$, which are consistent with our results that the anti-apoptosis protein Bcl-2 is related to mitochondria. Therefore, we validate that up-regulating Bcl-2 and down-regulating Bax by AR are connected with mitochondria-dependent cardioprotection in $\mathrm{H} / \mathrm{R}$-injured cardiomyocytes. However, the underlying mechanism is not fully clear and needs further study.

\section{Experimental Section}

\subsection{Materials}

Clematichinenoside (AR, 95.3\% purity, required concentration prepared with PBS before use) was prepared by the School of Traditional Chinese Pharmacy, China Pharmaceutical University (Nanjing, China). Foetal Bovine Serum (FBS) and Dulbecco's modified Eagle's medium (DMEM) were products of Hyclone Co. (Logan, UT, USA). Trypsin, penicillin and streptomycin were products of Grand Island Biological Company (Gibco, Grand Island, NY, USA). We purchased RIPA Buffer from Thermo Scientific Co. (Waltham, MA, USA); anti-Bcl-2 and anti-Bax from Cell Signaling Technology (Danvers, MA, USA); anti-cytochrome $c$ and anti-caspase- 3 from USCN Business Co. (Wuhan, China); anti- $\beta$-actin and the horseradish peroxidase-labeled IgG secondary antibodies from Boster Biological Technology (Wuhan, China). All other reagents were analytical grade and commercially available.

\subsection{Cell Culture}

The H9c2 cell line was provided by Boster Biological Technology and cultured with regular medium (DMEM with $15 \%$ FBS and $100 \mathrm{U} / \mathrm{mL}$ of penicillin and streptomycin) in humidified incubator of $5 \% \mathrm{CO}_{2}$ at $37{ }^{\circ} \mathrm{C}$. Two days later, $\mathrm{H} 9 \mathrm{c} 2$ cardiomyocytes grew into contractile layers, and the experiments of drug treatment were performed to induce the model of H9c2 cardiomyocytes hypoxia/reoxygenation (H/R) damage [16,31]. H9c2 cardiomyocytes were randomly and equally assigned into the five groups with different treatments. (1) Control group: No defined specific treatment; (2) H/R group: $5 \mathrm{mM} \mathrm{Na}_{2} \mathrm{~S}_{2} \mathrm{O}_{4}$ in the regular medium for $2 \mathrm{~h}$ (hypoxia) and then without $\mathrm{Na}_{2} \mathrm{~S}_{2} \mathrm{O}_{4}$ (reoxygenation) for $2 \mathrm{~h}$; (3) Low-dose $(1 \mu \mathrm{M})$ AR plus H/R group; (4) Intermediate-dose 
$(10 \mu \mathrm{M}) \mathrm{AR}$ plus H/R group; (5) High-dose $(100 \mu \mathrm{M}) \mathrm{AR}$ plus H/R group. The treatment duration of various doses of AR was $24 \mathrm{~h}$, and then the treated cardiomyocytes were washed twice with PBS before $\mathrm{H} / \mathrm{R}$ application.

\subsection{Detect Cell Viability by MTT Assay}

H9c2 cardiomyocytes were cultured and pretreated with the final concentrations of 1, 10, $100 \mu \mathrm{M}$ AR for $24 \mathrm{~h}$ prior to H/R treatment. After that, MTT $(0.5 \%, 20 \mu \mathrm{L})$ was added to the medium for $4 \mathrm{~h}$ at $37^{\circ} \mathrm{C}$ in the dark. The supernatant was removed, and dimethyl sulfoxide (DMSO, $100 \mu \mathrm{L} /$ well) was used to dissolve the precipitate. The absorbance was detected at $490 \mathrm{~nm}$ with a Multiskan Spectrum instrument (MK3, Thermo Scientific).

\subsection{Determination of Apoptosis by Flow Cytometry}

H9c2 cardiomyocytes were pretreated with concentrations of 1, 10, $100 \mu \mathrm{M}$ AR for $24 \mathrm{~h}$ before $\mathrm{H} / \mathrm{R}$ treatment, respectively. After centrifugation, cells $\left(1 \times 10^{6}\right)$ were resuspended in labeling solution for $15 \mathrm{~min}$ at room temperature in the dark. Cell fluorescence and apoptosis were analyzed by flow cytometry (Beckman ALTRA, Brea, CA, USA). Living cells, apoptotic cells in early or late phase of apoptosis were identified in quadrant dot plot. The Annexin V-FITC and PI (Roche, Los Angeles, CA, USA) double negative stained cells were identified as living cells. Only Annexin V-FITC positive cells were considered as early apoptotic cells and double positive staining cells were the late apoptosis cells. Apoptosis ratio was recognized as the percentage of Annexin V-FITC-positive cells.

\subsection{Determination of Apoptosis by TUNEL Assay}

To further observe the morphological difference between normal cells and apoptotic cells, apoptosis was also detected by TUNEL assay using an in situ cell death detection kit (Roche) according to the manufacturer's protocol. H9c2 cardiomyocytes were finally counterstained with DAPI (Sigma, St. Louis, MO, USA) for 5 min at room temperature, and examined using a TCS SP8 confocal laser scanning microscope (Leica, Solms, Germany). Four non-overlapping fields of vision were observed in each confocal dish. Apoptotic percentage was the ratio of TUNEL-positive nuclei to the total cell nuclei counterstaining by DAPI.

\subsection{The Opening of Mitochondrial Permeability Transition Pore (mPTP) Detection}

Transient mPTP opening was directly detected by using a mPTP assay kit (Genmed Scientifics Inc., Arlington, MA, USA) as described previously [32]. Briefly, in bottom 24 hole plate, H9c2 cardiomyocytes were rinsed with Reagent A, then treated with Reagents B and C (1:100; $500 \mu \mathrm{L} /$ well) at $37{ }^{\circ} \mathrm{C}$ for $20 \mathrm{~min}$ in the dark, after that rinsed twice in Reagent A. Fluorescent intensity was detected using a Varioskan Flash spectrofluorimeter (Thermo Scientific) at $\lambda_{\mathrm{ex}} 488 \mathrm{~nm}, \lambda_{\mathrm{em}} 505 \mathrm{~nm}$. Subsequently, cells were lysed and protein concentration was detected by Bradford Protein (Beyotime, Shanghai, China) assay. The fluorescence was normalized to total protein content. Results were represented as normalized relative fluorescence units (NRFU; $\mathrm{U} / \mathrm{mg}$ protein).

\subsection{Measurement of Mitochondrial Membrane Potential ( $\Delta \Psi m)$}

$\Delta \Psi \mathrm{m}$ of $\mathrm{H} 9 \mathrm{c} 2$ cardiomyocytes was measured by cationic probe JC-1 (Beyotime). JC-1 aggregates red fluorescence in mitochondria of normal cells. JC-1 accrues as green fluorescence monomer in the cytosol of apoptotic cells. $\Delta \Psi \mathrm{m}$ was increased linearly corresponds to the formation of JC-1 aggregates and their fluorescence [33]. Briefly, H9c2 cardiomyocytes were washed twice with PBS, and then loaded with JC- 1 at $37^{\circ} \mathrm{C}$ for $20 \mathrm{~min}$. After rinse twice in staining buffer, images were obtained using a Leica confocal laser scanning microscope (TCS SP8). Green and red fluorescent intensity was measured by Varioskan Flash (Thermo Scientific). The $\Delta \Psi \mathrm{m}$ was calculated as the fluorescent ratio of red vs. green, which indicated the $\Delta \Psi \mathrm{m}$ loss. 


\subsection{Analysis of Cytochrome c Leakage from Mitochondrial}

Cytochrome c level was determined in cytosol and isolated mitochondria. Isolated mitochondrial was prepared in accordance with instructions of the Mitochondria/Cytosol Isolation Kit (Sangon Biotech, Shanghai, China) as mentioned previously [34]. In brief, cells were collected and homogenized about 50 times with ice-cold Mito-Cyto Buffer in Dounce homogenizer. Following centrifugation, the supernatant was gathered and then centrifuged, which was cytosol protein. The precipitation was resuspended in Mito-Cyto Buffer to obtain the protein of mitochondrial. After Bradford protein assay, cytochrome c was analyzed by western blot (Figures S1-S4 of Supplementary Materials).

\subsection{Western Blot Analysis for Caspase-3, Bcl-2 and Bax}

H9c2 cardiomyocytes total proteins were prepared as depicted previously [35,36]. Cultured cells were rinsed twice in cold PBS and immersed in RIPA buffer on ice for $5 \mathrm{~min}$, and then lysates were gathered and centrifuged. Following protein concentration quantitation, denatured protein was separated by dodecyl sulfate sodium-polyacrylamide gel electrophoresis (SDS-PAGE) and then transferred to polyvinylidene difluoride (PVDF) membranes. PVDF membranes were blocked with $5 \%(w / v)$ non-fat milk for $1 \mathrm{~h}$ at $37^{\circ} \mathrm{C}$, and then incubated with primary antibodies of caspase-3, Bcl-2 and Bax overnight at $4^{\circ} \mathrm{C}$. After being rinsed in TBS-T three times, the membranes were incubated for $2 \mathrm{~h}$ with horseradish peroxidase combined secondary antibody. Images were analyzed using ChemiDoc XRS with Quantity One software (Bio-Rad, Hercules, CA, USA; Figures S5-S9 of the Supplementary Materials).

\subsection{Statistical Analysis}

Data were presented as mean \pm standard deviation (S.D) and analyzed with the GraphPad Prism 6.0 software. Comparisons between two groups were assessed using unpaired two-tailed Student's $t$-test. One-way ANOVA followed Bonferroni/Dunn post-hoc test was used for multiple comparisons. $p$ value $<0.05$ was considered statistically significant.

\section{Conclusions}

The present study illustrated that AR exerts cardioprotective effects hindering apoptosis by way of the mitochondrial signaling pathway against $H / R$ injury. This cardioprotective effect involves in decrease the extent of MPTP opening, inhibition of H/R-injured mitochondrial cytochrome c leakage, suppression of caspase-3 activity, and enhancement of the Bcl-2/Bax ratio. AR, via ameliorating the dysfunction of mitochondria, may become a promising drug to inhibit MI/R-induced apoptosis. Its apoptosis-inhibition may have important clinical application in MI/R injury. Further experiments are executed to validate the protective efficacy of AR in vivo and to provide potential clues for its clinical applications.

Supplementary Materials: Supplementary materials can be accessed at: http://www.mdpi.com/1420-3049/ $21 / 6 / 683 /$ s1.

Acknowledgments: This study was supported by the Natural Science Foundation of China Xinjiang Uygur Autonomous Region (2015211C001), Province Research Joint Innovation Fund-Prospective Joint Research Project of Jiangsu China (BY2014129) and 2012 Annual Doctoral Program of Higher Education Research Fund (20120096110004).

Author Contributions: H.D. participated in the design of the study and helped to draft the manuscript. H.R. participated in MTT, mPTP and flow cytometry detection. X.C. participated in TUNEL and JC-1 detection. W.F. helped to map the figures. M.L. performed the statistical analysis. X.W. participated in the western blot detection. Q.W. carried out the cell cultures. N.D.K. helped to fluent statements. Y.L. conceived of the study and coordination and participated in its design. All authors read and approved the final manuscript.

Conflicts of Interest: The authors declare no conflict of interest. 


\section{References}

1. Seif, A.A. Nigella sativa attenuates myocardial ischemic reperfusion injury in rats. J. Physiol. Biochem. 2013, 69, 937-944. [CrossRef] [PubMed]

2. Tehrani, D.M.; Seto, A.H. Third universal definition of myocardial infarction: update, caveats, differential diagnoses. Clevel. Clin. J. Med. 2013, 80, 777-786. [CrossRef] [PubMed]

3. Hausenloy, D.J.; Yellon, D.M. The evolving story of "conditioning" to protect against acute myocardial ischaemia-reperfusion injury. Heart 2007, 93, 649-651. [CrossRef] [PubMed]

4. Gottlieb, R.A.; Burleson, K.O.; Kloner, R.A.; Babior, B.M.; Engler, R.L. Reperfusion injury induces apoptosis in rabbit cardiomyocytes. J. Clin. Investig. 1994, 94, 1621-1628. [CrossRef] [PubMed]

5. Movassagh, M.; Foo, R.S. Simplified apoptotic cascades. Heart Fail. Rev. 2008, 13, 111-119. [CrossRef] [PubMed]

6. Konstantinidis, K.; Whelan, R.S.; Kitsis, R.N. Mechanisms of cell death in heart disease. Arterioscler. Thromb. Vasc. Biol. 2012, 32, 1552-1562. [CrossRef] [PubMed]

7. Sun, J.; Ling, Z.; Wang, F.; Chen, W.; Li, H.; Jin, J.; Zhang, H.; Pang, M.; Junjie, Y.U.; Liu, J. Clostridium butyricum pretreatment attenuates cerebral ischemia/reperfusion injury in mice via anti-oxidation and anti-apoptosis. Neurosci. Lett. 2015. [CrossRef] [PubMed]

8. Wu, M.-P.; Zhang, Y.-S.; Zhou, Q.-M.; Xiong, J.; Dong, Y.-R.; Yan, C. Higenamine protects ischemia/reperfusion induced cardiac injury and myocyte apoptosis through activation of ß2-AR/PI3K/AKT signaling pathway. Pharmacol. Res. 2016, 104, 115-123. [CrossRef] [PubMed]

9. Seidlmayer, L.K.; Blatter, L.A.; Pavlov, E.; Dedkova, E.N. Inorganic polyphosphate-An unusual suspect of the mitochondrial permeability transition mystery. Channels Austin Tex. 2012, 6, 463-467. [CrossRef] [PubMed]

10. Walter, D.H.; Haendeler, J.; Galle, J.; Zeiher, A.M.; Dimmeler, S. Cyclosporin A inhibits apoptosis of human endothelial cells by preventing release of cytochrome $C$ from mitochondria. Circulation 1998, 98, 1153-1157. [CrossRef] [PubMed]

11. Uchiyama, T.; Otani, H.; Okada, T.; Ninomiya, H.; Kido, M.; Imamura, H.; Nogi, S.; Kobayashi, Y. Nitric oxide induces caspase-dependent apoptosis and necrosis in neonatal rat cardiomyocytes. J. Mol. Cell. Cardiol. 2002, 34, 1049-1061. [CrossRef] [PubMed]

12. Shamas-Din, A.; Kale, J.; Leber, B.; Andrews, D.W. Mechanisms of action of Bcl-2 family proteins. Cold Spring Harb. Perspect. Biol. 2013, 5, a008714. [CrossRef] [PubMed]

13. Liu, L.F.; Ma, X.L.; Wang, Y.X.; Li, F.W.; Li, Y.M.; Wan, Z.Q.; Tang, Q.L. Triterpenoid saponins from the roots of Clematis chinensis Osbeck. J. Asian Nat. Prod. Res. 2009, 11, 389-396. [CrossRef] [PubMed]

14. Han, W.; Xiong, Y.; Li, Y.; Fang, W.; Ma, Y.; Liu, L.; Li, F.; Zhu, X. Anti-arthritic effects of clematichinenoside (AR-6) on PI3K/Akt signaling pathway and TNF-alpha associated with collagen-induced arthritis. Pharm. Biol. 2013, 51, 13-22. [CrossRef] [PubMed]

15. Peng, C.; Perera, P.K.; Li, Y.M.; Fang, W.R.; Liu, L.F.; Li, F.W. Anti-inflammatory effects of Clematis chinensis Osbeck extract(AR-6) may be associated with NF-kappaB, TNF-alpha, and COX-2 in collagen-induced arthritis in rat. Rheumatol. Int. 2012, 32, 3119-3125. [CrossRef] [PubMed]

16. Zhang, R.; Fang, W.; Han, D.; Sha, L.; Wei, J.; Liu, L.; Li, Y. Clematichinenoside attenuates myocardial infarction in ischemia/reperfusion injury both in vivo and in vitro. Planta Medica 2013, 79, 1289-1297. [CrossRef] [PubMed]

17. Javadov, S.; Karmazyn, M.; Escobales, N. Mitochondrial permeability transition pore opening as a promising therapeutic target in cardiac diseases. J. Pharmacol. Exp. Ther. 2009, 330, 670-678. [CrossRef] [PubMed]

18. Sarkey, J.P.; Chu, M.; McShane, M.; Bovo, E.; Ait Mou, Y.; Zima, A.V.; de Tombe, P.P.; Kartje, G.L.; Martin, J.L. Nogo-A knockdown inhibits hypoxia/reoxygenation-induced activation of mitochondrial-dependent apoptosis in cardiomyocytes. J. Mol. Cell. Cardiol. 2011, 50, 1044-1055. [CrossRef] [PubMed]

19. Ong, S.B.; Hall, A.R.; Hausenloy, D.J. Mitochondrial dynamics in cardiovascular health and disease. Antioxid. Redox Signal. 2013, 19, 400-414. [CrossRef] [PubMed]

20. Han, D.; Fang, W.; Zhang, R.; Wei, J.; Kodithuwakku, N.D.; Sha, L.; Ma, W.; Liu, L.; Li, F.; Li, Y. Clematichinenoside protects blood brain barrier against ischemic stroke superimposed on systemic inflammatory challenges through up-regulating A20. Brain Behav. Immun. 2016, 51, 56-69. [CrossRef] [PubMed] 
21. Galluzzi, L.; Morselli, E.; Kepp, O.; Kroemer, G. Targeting post-mitochondrial effectors of apoptosis for neuroprotection. Biochim. Biophys. Acta 2009, 1787, 402-413. [CrossRef] [PubMed]

22. Di Lisa, F.; Canton, M.; Menabo, R.; Kaludercic, N.; Bernardi, P. Mitochondria and cardioprotection. Heart Fail. Rev. 2007, 12, 249-260. [CrossRef] [PubMed]

23. Yue, R.; Hu, H.; Yiu, K.H.; Luo, T.; Zhou, Z.; Xu, L.; Zhang, S.; Li, K.; Yu, Z. Lycopene protects against hypoxia/reoxygenation-induced apoptosis by preventing mitochondrial dysfunction in primary neonatal mouse cardiomyocytes. PLoS ONE 2012, 7, e50778. [CrossRef] [PubMed]

24. Yao, K.; Ye, P.; Zhang, L.; Tan, J.; Tang, X.; Zhang, Y. Epigallocatechin gallate protects against oxidative stress-induced mitochondria-dependent apoptosis in human lens epithelial cells. Mol. Vis. 2008, 14, 217-223. [PubMed]

25. Zhang, T.; Saghatelian, A. Emerging roles of lipids in BCL-2 family-regulated apoptosis. Biochim. Biophys. Acta BBA Mol. Cell Biol. Lipids 2013, 1831, 1542-1554. [CrossRef] [PubMed]

26. Kumar, D.; Jugdutt, B.I. Apoptosis and oxidants in the heart. J. Lab. Clin. Med. 2003, 142, 288-297. [CrossRef]

27. Lee, Y.; Gustafsson, A.B. Role of apoptosis in cardiovascular disease. Apoptosis 2009, 14, 536-548. [CrossRef] [PubMed]

28. Borutaite, V.; Brown, G.C. Mitochondria in apoptosis of ischemic heart. FEBS Lett. 2003, 541, 1-5. [CrossRef]

29. Cook, S.A.; Sugden, P.H.; Clerk, A. Regulation of bcl-2 family proteins during development and in response to oxidative stress in cardiac myocytes: Association with changes in mitochondrial membrane potential. Circ. Res. 1999, 85, 940-949. [CrossRef] [PubMed]

30. Nishihara, M.; Miura, T.; Miki, T.; Tanno, M.; Yano, T.; Naitoh, K.; Ohori, K.; Hotta, H.; Terashima, Y.; Shimamoto, K. Modulation of the mitochondrial permeability transition pore complex in GSK-3beta-mediated myocardial protection. J. Mol. Cell. Cardiol. 2007, 43, 564-570. [CrossRef] [PubMed]

31. Fang, W.; Deng, Y.; Li, Y.; Shang, E.; Fang, F.; Lv, P.; Bai, L.; Qi, Y.; Yan, F.; Mao, L. Blood brain barrier permeability and therapeutic time window of Ginkgolide B in ischemia-reperfusion injury. Eur. J. Pharm. Sci. 2010, 39, 8-14. [CrossRef] [PubMed]

32. Petronilli, V.; Miotto, G.; Canton, M.; Brini, M.; Colonna, R.; Bernardi, P.; Di Lisa, F. Transient and long-lasting openings of the mitochondrial permeability transition pore can be monitored directly in intact cells by changes in mitochondrial calcein fluorescence. Biophys. J. 1999, 76, 725-734. [CrossRef]

33. Smiley, S.T.; Reers, M.; Mottola-Hartshorn, C.; Lin, M.; Chen, A.; Smith, T.W.; Steele, G.D., Jr.; Chen, L.B. Intracellular heterogeneity in mitochondrial membrane potentials revealed by a J-aggregate-forming lipophilic cation JC-1. PNAS 1991, 88, 3671-3675. [CrossRef] [PubMed]

34. Li, H.Z.; Guo, J.; Gao, J.; Han, L.P.; Jiang, C.M.; Li, H.X.; Bai, S.Z.; Zhang, W.H.; Li, G.W.; Wang, L.N.; et al. Role of dopamine D2 receptors in ischemia/reperfusion induced apoptosis of cultured neonatal rat cardiomyocytes. J. Biomed. Sci. 2011, 18. [CrossRef] [PubMed]

35. Wang, L.N.; Wang, C.; Lin, Y.; Xi, Y.H.; Zhang, W.H.; Zhao, Y.J.; Li, H.Z.; Tian, Y.; Lv, Y.J.; Yang, B.F.; et al. Involvement of calcium-sensing receptor in cardiac hypertrophy-induced by angiotensinII through calcineurin pathway in cultured neonatal rat cardiomyocytes. Biochem. Biophys. Res. Commun. 2008, 369, 584-589. [CrossRef] [PubMed]

36. Wang, L.; Zheng, X.; Xiang, H.L.; Fu, X.H.; Cao, J.G. 7-Difluoromethyl-5,4'-dimethoxygenistein inhibits oxidative stress induced adhesion between endothelial cells and monocytes via NF-kappaB. Eur. J. Pharmacol. 2009, 605, 31-35. [CrossRef] [PubMed]

Sample Availability: Samples of the compounds clematichinenoside (AR) are available from the authors. 